\title{
Saikosaponin-D Enhances Radiosensitivity of Hepatoma Cells under Hypoxic Conditions by Inhibiting Hypoxia-Inducible Factor-1 $\alpha$
}

\author{
Bao-Feng Wang ${ }^{a, c}$ Xi-jing Wang ${ }^{a}$ Hua-Feng Kanga,c Ming-Hua Baia \\ Hai-Tao Guan ${ }^{a}$ Zhong-Wei Wang ${ }^{a}$ Ying Zan ${ }^{a} \quad$ Ling-Qin Song $^{a} \quad$ Wei-Li Mina \\ Shuai Lin ${ }^{a}$ Yan-An Cheng ${ }^{b}$ \\ aDepartment of Oncology, the Second Affiliated Hospital of School of Medicine, Xi'an Jiaotong \\ University, Xi'an, 'Department of Infectious Disease, the Second Affiliated Hospital of School of \\ Medicine, Xi'an Jiaotong University, Xi'an, P.R. China; 'Co-first authors
}

\section{Key Words}

Hypoxia • HCC • Radiation • Cobalt chloride • HIF-1 $\alpha$

\begin{abstract}
Background: Our previous study revealed that the combination of Saikosaponin-d (SSd) and radiation is more effective in the treatment of liver cancer than the application of either of these monotherapeutic methods. However, the molecular mechanisms of the radiosensitizing effect of SSd on liver cancer remained ill defined. Methods: Cells were treated with different interventions; afterward, cell viability, apoptosis, and cell survival of SMMC-7721 and HepG2 hepatoma cells were examined. Xenograft tumor models were established by subcutaneously injecting SMMC-7721 cells. The molecular mechanism was assessed by western blot. Results: SSd dose-dependently increased radiosensitivity of hepatoma cells under hypoxic condition. The growth inhibitory effect of the combined treatment was correlated with cell apoptosis. Further mechanistic analysis indicated that SSd induced the upregulation of p53 and Bax as well as the downregulation of $\mathrm{BCl}-2$ by attenuating HIF-1 $\alpha$ expression under hypoxic condition. These effects were enhanced when the HIF-1 $\alpha$ inhibitor PX-478 was introduced. In vivo data also presented a more significant suppression of tumor xenograft growth from the combined therapy than from either of the monotherapeutic methods. Conclusions: Our study provides evidence for a radiosensitizing effect of SSd on hepatoma cells under hypoxic conditions by inhibiting HIF-1 $\alpha$ expression. Thus, SSd can be used as a potential sensitizer in hepatoma radiotherapy.
\end{abstract}


Wang et al.: Saikosaponin-d Enhances Radiosensitivity by Inhibiting HIF-1 $\alpha$

\section{Introduction}

Hepatocellular carcinoma (HCC) is one of the most common malignant tumors worldwide; the incidence of HCC has increased for several decades [1-3]. In many cases, HCC is developed from viral hepatitis and cirrhosis. Although many treatment strategies, such as surgical resection, liver transplantation, radiotherapy, and chemotherapy, have been introduced, patient survival has not evidently improved. This problem occurs because HCC has reached an advanced stage at the time of clinical presentation; therapeutic options at this stage are very limited. In recent years, new technologies, including three-dimensional conformal radiotherapy and intensity modulated radiation therapy, have been used to treat patients with advanced HCC [4-6]. However, the presence of hypoxic tumor cells that are more invasive, metastatic, and radioresistant influences radiotherapeutic outcomes [7, 8].

Hypoxia is a common characteristic of rapidly growing malignant tumors that promote cancer progression, cause resistance to radiotherapy and chemotherapy, and predict poor overall patient survival. Hypoxia-inducible factor- $1 \alpha$ (HIF- $1 \alpha$ ) in tumor cells can increase radiation resistance and tumor angiogenesis $[9,10]$; HIF-1 $\alpha$ expression can be induced by radiation [11]. Studies have demonstrated that HIF-1 $\alpha$ expression in tumor cells can be inhibited; as a result, tumor angiogenesis may be reduced and tumor radiosensitivity may be enhanced, thereby inhibiting the growth of xenografted tumors [11-13].

Saikosaponin-d (SSd), an extract from a traditional Chinese herb, Bupleurum chinensis DC, exhibits various pharmacological properties, such as anti-inflammation $[14,15]$, hepatocyte injury prevention [16], angiogenesis inhibition [17], and anti-cancer [18, 19]. In our preliminary experiments, SSd and radiation are administered to treat liver cancer; the results have revealed that this combined treatment is more effective than either of these treatments administered alone. This result has indicated that SSd contributes to the effects of radiotherapy. We also observed that SSd elicits anti-cancer effect at a low extent, but SSd remarkably enhances the radiosensitivity of tumor cells when used at a very low concentration. Considering that HIF-1 $\alpha$ expression in tumor cells is a key factor of radioresistance, we assumed that SSd may increase radiosensitivity by inhibiting HIF$1 \alpha$-active hypoxic tumor cells. To verify this hypothesis, we established an in vitro $\mathrm{CoCl}_{2}$ induced hepatoma hypoxia model and an in vivo mouse xenograft tumor model. We used these models to investigate the response of hepatoma to SSd when administered alone or combined with radiation.

\section{Materials and Methods}

\section{Reagents and antibodies}

PX-478-, HIF-1 $\alpha$-, and VEGF-specific monoclonal antibodies were purchased from Millipore Co. (USA). SSd and $\mathrm{CoCl}_{2}$ were obtained from Sigma Chemical (St. Louis, MO). Hypoxyprobe ${ }^{\mathrm{TM}}-1$ Kit was supplied by Hypoxyprobe Inc. (Burlington, MA). P53, Bax, Bcl-2, and $\beta$-actin antibodies were obtained from Santa Cruz Biotechnology (Santa Cruz, CA).

\section{Cell culture and hypoxia model}

Two hepatoma cell lines, SMMC-7721 and HepG2, were purchased from the Medical Experimental Animal Center of the Fourth Military Medical University (Xi'an, China). The cells were cultured in RPMI1640 medium (PAA Laboratories GmbH, Austria) supplemented with 10\% fetal bovine serum (FBS), 100 units $/ \mathrm{ml}$ penicillin $\mathrm{G}$, and $100 \mu \mathrm{g} / \mathrm{ml}$ streptomycin sulfate (GIBCO, Invitrogen) in a humidified atmosphere containing $5 \% \mathrm{CO}_{2}$ at $37{ }^{\circ} \mathrm{C}$. These cells grown to $80 \%$ to $90 \%$ confluence were trypsinized, counted, and seeded in a six-well plate to induce hypoxia. The cells were incubated with $100 \mu \mathrm{M} \mathrm{CoCl}_{2}$ for $4 \mathrm{~h}$ in $4 \mathrm{ml}$ of serum-free medium and then exposed to X-ray irradiation at a dose rate of $400 \mathrm{cGy} / \mathrm{min}$ (Clinac 2100EX; Varian Medical Systems Inc., CA) [20, 21]. Immediately after irradiation, the medium was replaced with fresh RPMI-1640 containing 10\% FBS and the cells were incubated at $37{ }^{\circ} \mathrm{C}$ for $24 \mathrm{~h}$ before cell viability was determined. In addition, hypoxia was made by placing cells in a microaerophilic incubation system 
(Bugbox-M, Ruskinn, Bridgend, UK) with humidified $1 \% \mathrm{O}_{2}, 5 \% \mathrm{CO}_{2}$, and $94 \% \mathrm{~N}_{2}$. The control cultures were incubated in oxic conditions of humidified $21 \% \mathrm{O}_{2}$.

\section{SSd preparation and experimental groups}

SSd was dissolved in dimethylsulfoxide (DMSO, Sigma, St. Louis, MO, USA) and stored at $-20^{\circ} \mathrm{C}$. The desired final concentrations were obtained by diluting with RPMI-1640. SMMC-7721 and HepG2 cells were treated with radiation alone, SSd alone, or a combination of radiation and SSd. Radiation was performed at different doses $(2,4,6$, and $8 \mathrm{~Gy}, 6 \mathrm{MV}$, and a dose rate of $400 \mathrm{cGy} / \mathrm{min})$ by using an X-ray linear accelerator at room temperature. SSd was also administered at different concentrations $(1,3,5,10$, and $20 \mu \mathrm{g} / \mathrm{ml})$. SSd was added to the cultures at $2 \mathrm{~h}$ before irradiation. Control cultures received a carrier solvent consisting of $0.1 \%$ DMSO.

\section{Cell viability determination}

Cell viability was detected by MTT assay under oxic condition or $\mathrm{CoCl}_{2}$-induced hypoxic condition after $4 \mathrm{~h}$ of chemical hypoxic culture. SMMC-7721 and HepG2 cells were seeded in a 96-well plate $\left(5 \times 10^{3}\right.$ cells/well) and incubated at $37{ }^{\circ} \mathrm{C}$ in $5 \% \mathrm{CO}_{2}$ for different periods as desired. MTT solution $(5 \mathrm{mg} / \mathrm{ml}$; Sigma, St. Louis, MO, USA) was added ( $20 \mu \mathrm{l} /$ well $)$ and the cells were incubated for another $4 \mathrm{~h}$. Supernatants were removed and formazan crystals were dissolved in $200 \mu \mathrm{l}$ of DMSO. Optical density was determined at 492 $\mathrm{nm}$ by using a multi-microplate test system (POLARstar OPTIMA, BMG Labtechnologies, Germany). The assay was conducted in quadruplicate.

\section{Cell survival analysis}

The cells in the monolayer culture were exposed to SSd or $0.1 \%$ DMSO for 24 or $48 \mathrm{~h}$ and irradiated with graded doses of X-ray by using a linear accelerator at a dose rate of $400 \mathrm{cGy} / \mathrm{min}$. The cells that remained attached were removed from the culture flask by exposing to $0.05 \%$ trypsin/ $1 \mathrm{mM}$ EDTA solution. Afterward, these cells were re-plated in specified numbers in $60 \mathrm{~mm}$ dishes containing a drug-free medium to determine the colony-forming ability. After $10 \mathrm{~d}$ of incubation, the dishes were stained with $0.5 \%$ crystal violet in absolute methanol, and the colonies with more than 50 cells were counted. The colony-forming rate was defined as the number of colonies divided by the number of cells seeded at 0 Gy. The survival fraction (SF) was calculated by dividing the number of colonies at a certain radiation dose by the product of the number of cells seeded at this dose and the corresponding colony-forming rate. The sensitization enhancement ratio (SER) at 50\% cell survival was obtained by dividing the dose of radiation from the radiation-only surviving curve by the corresponding dose from the SSd plus radiation curve. Each data point was calculated as the percent of untreated cells in two independent experiments each performed in duplicates.

\section{Apoptosis assay}

In vitro cell apoptosis was detected by flow cytometry using an Annexin V-FITC apoptosis detection kit (BD Bioscience Co., USA,). In brief, the cells in the tumor samples were double stained with Annexin V-FITC and propidium iodide (PI) according to the manufacturer's instructions. Early apoptosis and late apoptosis were determined by Annexin V+/PI- staining (Q4) and Annexin V+/PI+ staining (Q2), respectively. The percentage of apoptotic cells in each sample was examined using a FACSCalibur ${ }^{\mathrm{TM}}$ flow cytometer (Becton Dickinson Technologies, USA) and compared with the control samples.

To determine cell apoptosis in vivo, $5 \mu \mathrm{m}$ tissue sections were deparaffinized in xylene and hydrated in decreasing ethanol concentrations. Terminal deoxynucleotidyl transferase-mediated dUTP nick end labeling (TUNEL) assay was performed according to the manufacturer's instructions (Keygen, Nanjing, China). A total of 10 random fields from four slides per group were examined. TUNEL-positive brown nuclei in the tissue were counted. Data were expressed as the mean number of apoptotic cells divided by the number of high power fields.

\section{Xenograft tumor model and treatment}

A therapeutic study was conducted on SMMC-7721 xenograft tumor models. Male BALB/c nude mice (age $=$ four weeks) were purchased from the Experimental Animal Center of School of Medicine, Xi'an Jiaotong University (Xi'an, China). The tumor model was established by subcutaneously (s.c.) injecting $2 \times$ 
$10^{6}$ SMMC-7721 cells $(0.2 \mathrm{~mL})$ in the left leg of each mouse. After the tumors reached a mean volume of 300 $\mathrm{mm}^{3}$ to $400 \mathrm{~mm}^{3}$, the mice were randomly distributed into four groups $(n=6)$ : SSd, radiation, combined, and control groups. The mice in the SSd group were intraperitoneally injected with SSd at a dose of 0.75 $\mathrm{mg} / \mathrm{kg}$ body weight thrice a week for two weeks. This dose was administered based on the result of our preliminary experiment, which is $10 \%$ of the dose with the median effective concentration $\left(\mathrm{EC}_{50}\right)$ of SSd when administered alone. The mice treated by radiation alone were immobilized in a customized harness. The right leg was exposed and the remaining parts of the body were shielded with a thick $(7 \mathrm{~cm})$ piece of lead. The tumor area was irradiated with a single dose of $10 \mathrm{~Gy}$ at a dose rate of $500 \mathrm{cGy} / \mathrm{min}$ by using an X-ray linear accelerator. The mice in the group treated with the combined SSd and radiation were irradiated with $10 \mathrm{~Gy}$ at $24 \mathrm{~h}$ after the third injection of SSd in the first week. The control group received a vehicle consisting of $0.1 \%$ DMSO.

Tumor growth was monitored by measuring the tumor size in two orthogonal dimensions with vernier calipers every $5 \mathrm{~d}$; the tumor volume was calculated according to the following formula: [length $x$ (width2)] $\times 0.5[22,23]$. The mice were anesthetized and sacrificed three weeks after the final treatment. The tumors were surgically excised, weighed, fixed in $10 \%$ formalin, embedded in paraffin, and cut into thick slices $(4 \mu \mathrm{m})$ for hematoxylin and eosin staining (H\&E). Inhibition rate was defined as: [1- tumor weight or volume of the treatment group / tumor weight or volume of the control group]. In addition, the tumor-bearing mice were intraperitoneally injected with pimonidazole hydrochloride $(60 \mathrm{mg} / \mathrm{kg})$, and the solid tumors were then surgically removed and fixed in $10 \%$ formalin neutral buffer solution after $60 \mathrm{~min}$. Immunohistochemical staining of pimonidazole was performed according to a standard protocol using a Hypoxyprobe ${ }^{\mathrm{TM}}-1$ kit. The primary antibodies to pimonidazole (1:100) were selected. PBS, instead of primary antibody, was used as a negative control. Pimonidazole positive staining was judged according to the methods provided by Nordsmark [24]. Data were representative of three independent experiments. All of the animal procedures were conducted in accordance with the Guide for the Care and Use of Laboratory Animals of the National Institutes of Health. Experimental protocols were approved by the Animal Care and Use Regulation of Xi'an Jiaotong University.

\section{Western blot}

Western blot analysis was performed as described previously [23]. In brief, aliquots of cell lysates containing $40 \mu \mathrm{g}$ of protein were separated on 10\% SDS-PAGE gels and transferred to nitrocellulose membranes (Millipore Billerica, MA). The blots were probed with antibodies against HIF-1 $\alpha$ (diluted 1:200), VEGF (diluted 1:200), p53 (diluted 1:100), Bcl-2 (diluted 1:100), and Bax (diluted 1:100). The membranes were incubated in the dark with ECL (Amersham) to detect chemiluminescence. Luminescent signals were detected using a CCD camera, recorded, and quantified using a Syngene G Box (Syngene, UK). The membranes were re-probed for $\beta$-actin as loading control. Analysis was conducted at least thrice for each experiment.

\section{Statistical analysis}

Quantitative data were presented as the mean \pm standard error of the mean (SEM) and analyzed by one-way ANOVA. Statistical analyses were performed using SPSS software (version 13.0). Tukey's post hoc analyses were conducted to assess the difference between groups. Data were considered significant if $P<$ 0.05 .

\section{Results}

Effect of SSd on cell growth and apoptosis

The chemical structure of SSd is shown in Fig. 1A. Cell growth in response to SSd treatment was assessed under oxic or hypoxic condition. Cell viability was determined at 48 $\mathrm{h}$ after treatment. Fig. 1B shows that the cell viabilities of HepG2 and SMMC-7721 cells were significantly reduced in a dose-dependent manner under oxic and $\mathrm{CoCl}_{2}$ conditions. $\mathrm{IC}_{50}$ was $13.2 \mu \mathrm{g} / \mathrm{ml}$ for SMMC-7721 and $17.7 \mu \mathrm{g} / \mathrm{ml}$ for HepG2 cells under oxic conditions; IC $_{50}$ was $14.3 \mu \mathrm{g} / \mathrm{ml}$ for SMMC-7721 and $19.7 \mu \mathrm{g} / \mathrm{ml}$ for HepG2 under $\mathrm{CoCl}_{2}$ conditions.

Cell apoptosis induced by SSd, radiation, or both was determined by flow cytometry. PX-478 was used as a positive control. The results showed that $\geq 3 \mu \mathrm{g} / \mathrm{ml}$ of SSd significantly 
Fig. 1. Growth-inhibitory effects of SSd in two cancer cell lines. A. Chemical structure of SSd from drug instructions. Molecular weight was 780.98. B. MTT assay of the growth of HepG2 and SMMC-7721 cells treated with SSd for $48 \mathrm{~h}$ under oxic (N) and $\mathrm{CoCl}_{2}(\mathrm{H})$ conditions. IC50 ( $\mu \mathrm{g} /$ $\mathrm{ml})$ was $17.7(\mathrm{~N})$ and $19.7(\mathrm{H})$ for HepG2 cells and $13.2(\mathrm{~N})$ and $14.3(\mathrm{H})$ for SMMC-7721 cells. $\mathrm{C}$. Effects of SSd, radiation, and PX478 alone or their combination on SMMC-7721 cell apoptosis under oxic and $\mathrm{CoCl}_{2}$ conditions. D. Effects of SSd, radiation, and PX-478 alone or their combination on HepG2 cell apoptosis under oxic and $\mathrm{CoCl}_{2}$ conditions. E. Effects of SSd and radiation, alone or in combination, on SMMC7721 cell apoptosis under oxic and hypoxic conditions. F. Effects of SSd and radiation, alone or in combination, on HepG2 cell apoptosis under oxic and hypoxic conditions. Cell apoptosis evaluated by flow cytometry using annexin-V/PI staining revealed a marked increase in apoptosis in cells treated with joint therapies especially under $\mathrm{CoCl}_{2}$ or hypoxic condition. Data are shown as mean \pm SD ${ }^{*} \mathrm{P}<0.05,{ }^{* *} \mathrm{P}<0.01$.

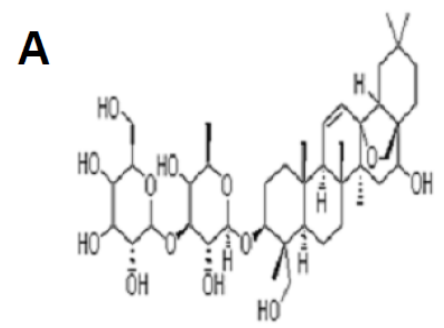

Saikosaponin-d-(C42H68013)
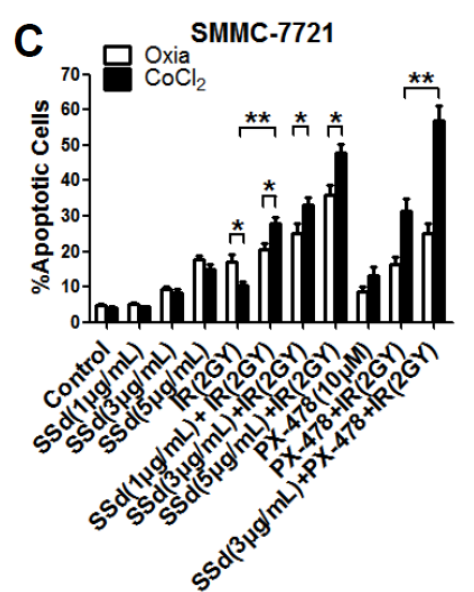

E

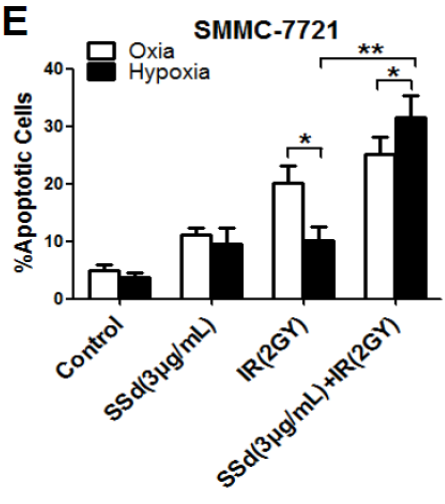

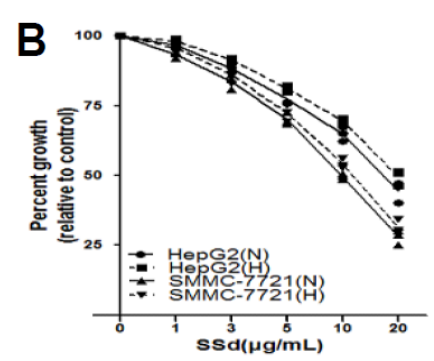

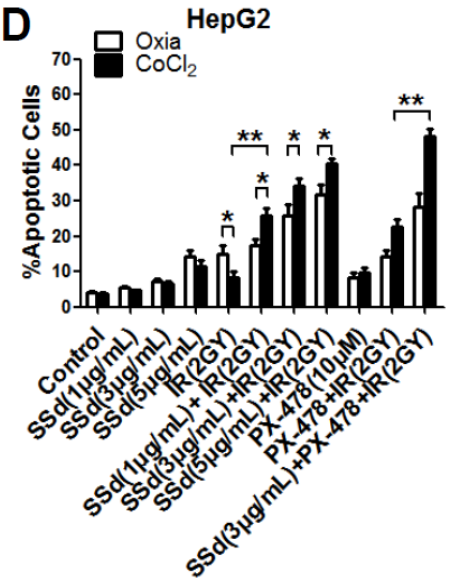

$\mathbf{F}$

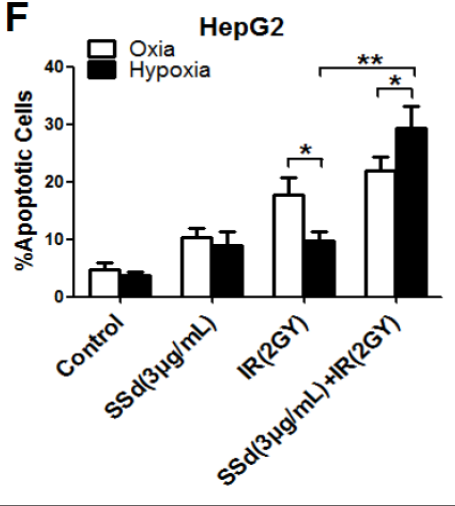

induced apoptosis of the two cell lines (Figs. 1C and 1D) under oxic and $\mathrm{CoCl}_{2}$ conditions compared with the control cells $(P<0.05)$. Induced apoptosis increased in both cell lines under oxic condition compared with the cells under $\mathrm{CoCl}_{2}$ condition, but the difference was not significant (Figs. 1C and 1D, $P>0.05$ ). At 2 Gy of radiation, the percentage of apoptotic cells under oxic condition was higher than that under $\mathrm{CoCl}_{2}$ condition in both cell lines (Figs. 1C and 1D, $P<0.05$ ). The combined therapy induced the apoptosis of both cell lines at a greater extent under both oxic and $\mathrm{CoCl}_{2}$ conditions than either SSd or radiation therapy alone. SSd did not induce apoptosis in the cell lines when used alone at a low dose $(1 \mu \mathrm{g} /$ $\mathrm{ml}$ ). By contrast, the combined treatment at the same concentration exhibited a synergistic effect on apoptosis induction. In SMMC-7721 cell line, the apoptosis rates under $\mathrm{CoCl}_{2}$ condition were $10.46 \%$ in the radiation group and $27.85 \%$ in the combined treatment group (Fig. 1C, $P<0.01$ ). A similar change was observed in HepG2 cell line, in which the apoptosis rates were $8.37 \%$ and $25.78 \%$ in radiation and combined treatment groups, respectively (Fig. 1D, $P<0.01$ ). The synergistic effect of SSd on radiation-induced apoptosis was more evident under $\mathrm{CoCl}_{2}$ condition than under oxic condition. This effect was observed in a dose- 


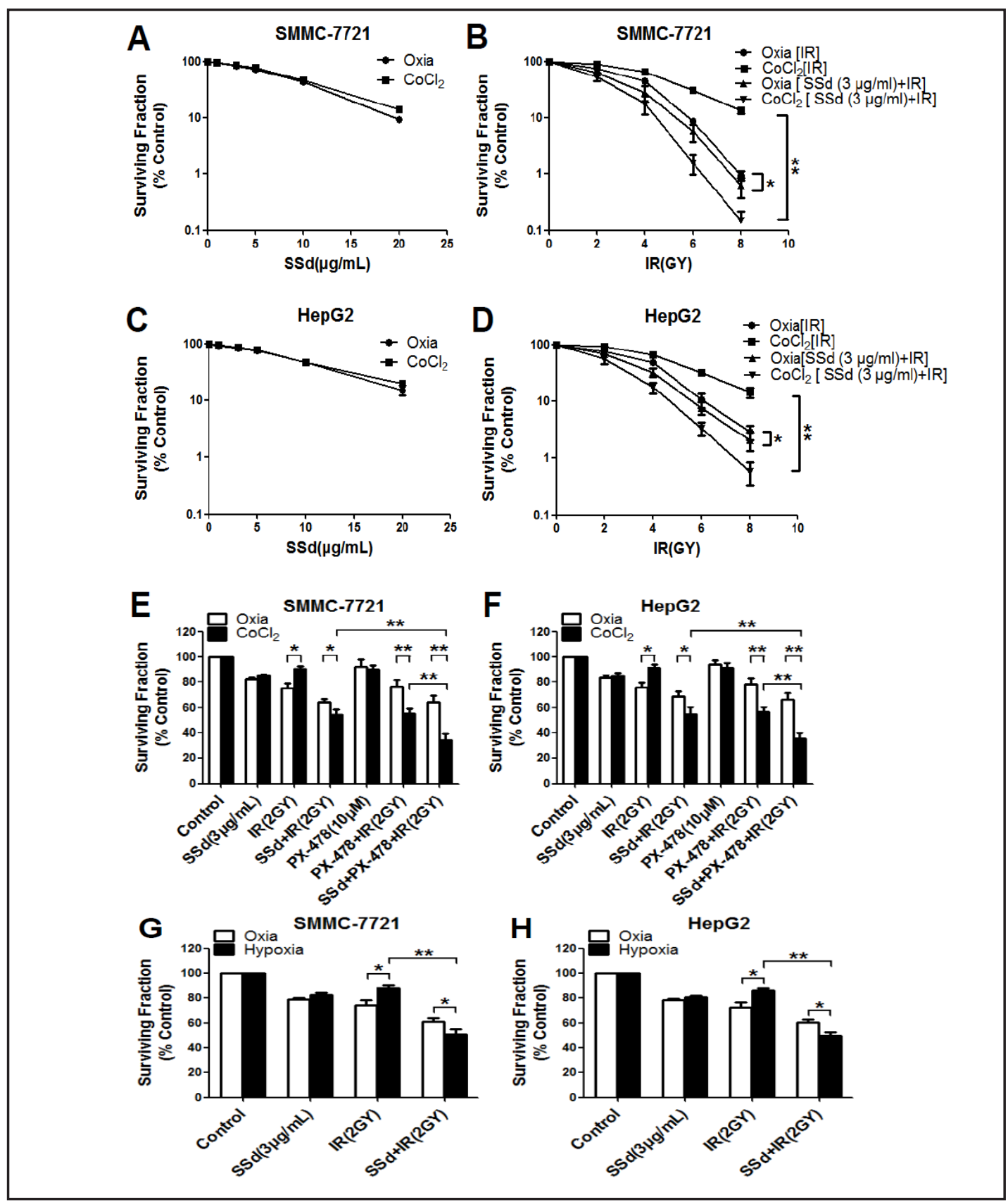

Fig. 2. Survival curves of SMMC-7721 and HepG2 cells exposed to different interventions. A. C. SSd alone at different concentrations. B. D. Radiation alone and combined effects of radiation with SSd $(3 \mu \mathrm{g} / \mathrm{ml})$. E. F. Surviving fractions of the two cell lines exposed to different interventions. G. Surviving fractions of the SMMC-7721 cell under oxic or hypoxic condition. H. Surviving fractions of the HepG2 cell under oxic or hypoxic condition. The survival curves show a pronounced decrease in cell colony formation and an evident increase in cell radiosensitivity especially under $\mathrm{CoCl}_{2}$ or hypoxic condition, and the effect appeared to be dose-dependent. The surviving fraction was determined as a percentage with respect to control cells. Data are shown as mean $\pm \mathrm{SD} * \mathrm{P}<0.05,{ }^{* *} \mathrm{P}<0.01$.

dependent manner (Figs. 1C and 1D, $P<0.05$ ). PX-478 further enhanced this effect under $\mathrm{CoCl}_{2}$ condition. Similarly, the synergistic effect of SSd on radiation-induced apoptosis was also observed under hypoxic conditions $\left(1 \% 02,5 \% \mathrm{CO}_{2}\right.$, and $94 \% \mathrm{~N}_{2}$ ) (Figs. $1 \mathrm{E}$ and $1 \mathrm{~F}$, $P<0.01)$. 


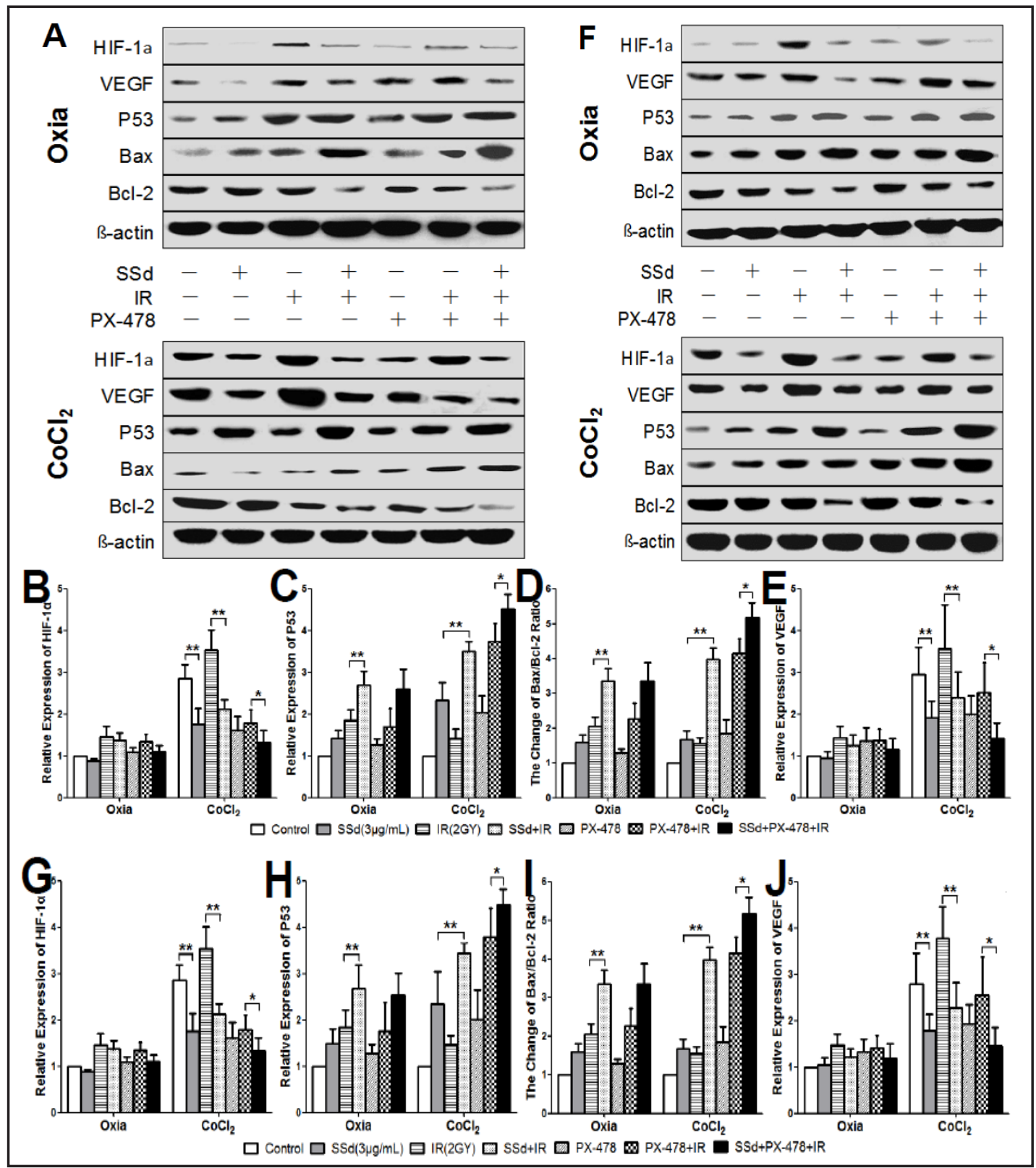

Fig. 3. Effects of different interventions on the expressions of HIF-1 $\alpha$, VEGF, P53, Bax and Bcl-2 in the two cell lines. Cells were treated with vehicle control (0.1\% DMSO), SSd or radiation alone, or the combination of SSd and radiation under oxia or $\mathrm{CoCl}_{2}$. $\beta$-actin was used to verify equal loading of the protein samples on the gel. A-E. SMMC-7721 cells. A. Western Blotting of HIF-1 $\alpha$, VEGF, p53, Bax and Bcl-2 levels. B. Relative expression of HIF-1 $\alpha$. C. Relative expression of p53. D. Change of Bax/Bcl-2 ratio. E. Relative expression of VEGF. F-J. HepG2 cells. F. Western Blotting of HIF-1 $\alpha$, VEGF, p53, Bax and Bcl-2 levels. G. Relative expression of HIF-1 $\alpha$. H. Relative expression of p53. I. Change of Bax/Bcl-2 ratio. J. Relative expression of VEGF. Data are shown as mean $\pm \mathrm{SD} * \mathrm{P}<0.05, * * \mathrm{P}<0.01$.

\section{Effect of SSd on cell colony formation}

SMMC-7721 and HepG2 cell lines were treated with SSd, radiation, or a combination of SSd ( $3 \mu \mathrm{g} / \mathrm{mL})$ and radiation $(2,4,6$, and $8 \mathrm{~Gy})$. Untreated cells were used as the control. The cells were re-plated at certain dilutions after treatment and the surviving colonies were counted after $10 \mathrm{~d}$. A more evident, dose-dependent decrease in cell survival was observed in the combined treatment group than in SSd or radiation group, particularly under $\mathrm{CoCl}_{2}$ 


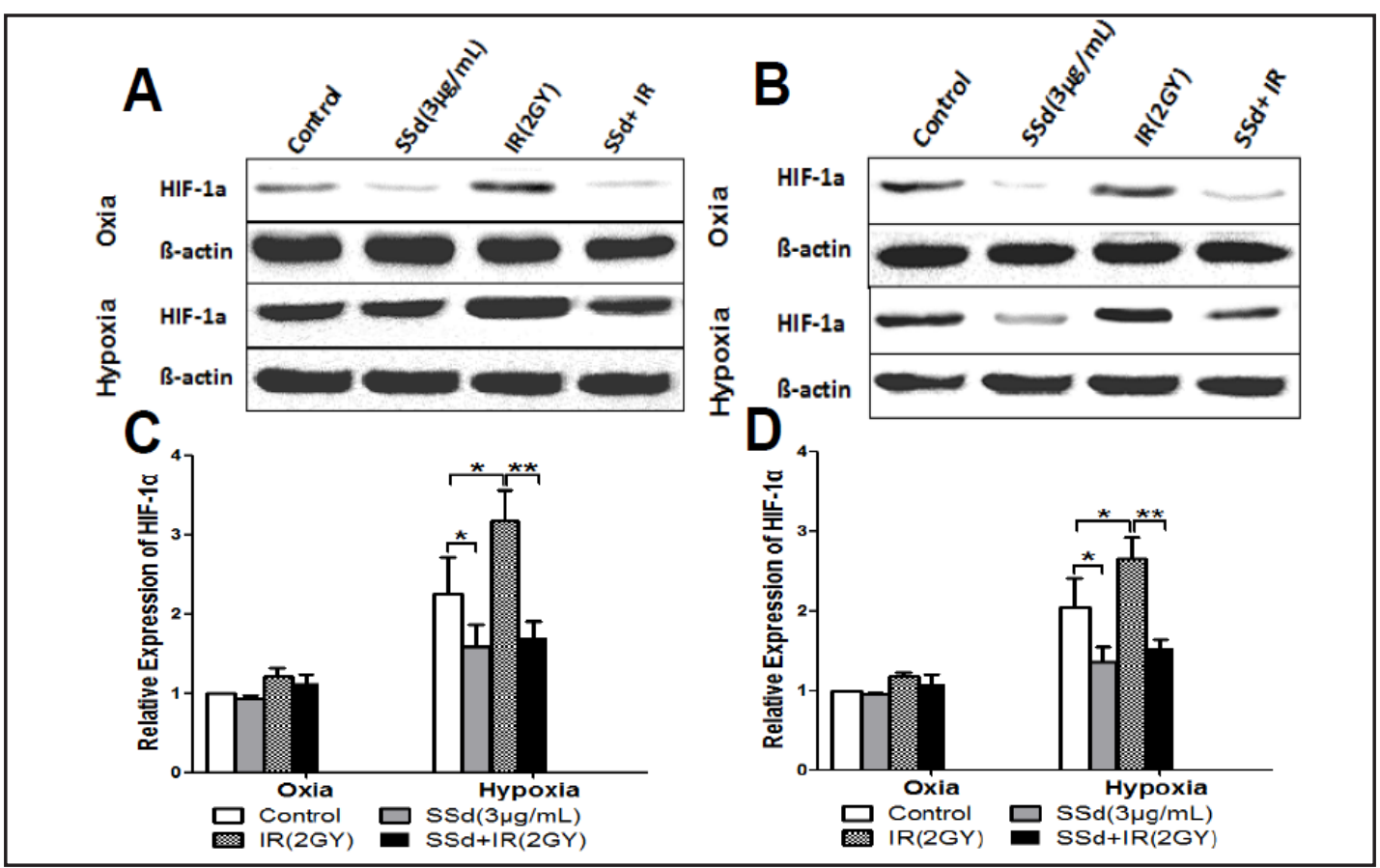

Fig. 4. Effects of different interventions on HIF-1 $\alpha$ expression in the two cell lines. Cells were treated with SSd or radiation alone, or with the combination of SSd and radiation under oxic or hypoxic condition. A. Western blot analysis of the HIF-1 $\alpha$ levels in SMMC-7721 cells. B. Western blot analysis of HIF-1 $\alpha$ levels in HepG2 cells. C. Relative HIF-1 $\alpha$ expression in SMMC-7721 cells. D. Relative HIF-1 $\alpha$ expression in HepG2 cells. Data are shown as mean $\pm \mathrm{SD} * \mathrm{P}<0.05,{ }^{* *} \mathrm{P}<0.01$.

condition (Fig. 2; $P<0.01$ ). SFs of SMMC-7721 cells were 0.75 and 0.91 under oxic and $\mathrm{CoCl}_{2}$ conditions, respectively, when the cells were exposed to 2 Gy of radiation (Fig. 2B). SFs of HepG2 cells were 0.76 and 0.92 under oxic and $\mathrm{CoCl}_{2}$ conditions, respectively (Fig. 2D). This result indicated that radiation alone did not effectively inhibit the cell survival under $\mathrm{CoCl}_{2}$ condition as observed under oxic condition. After SSd ( $\left.3 \mu \mathrm{mol} / \mathrm{L}\right)$ was combined with radiotherapy, SF of SMMC-7721 cells at $2 \mathrm{~Gy}$ of radiation decreased to 0.54 under $\mathrm{CoCl}_{2}$ condition (Fig. 2B). SF of HepG2 cells also decreased to 0.55 (Fig. 2D). SERs were 1.72 and 1.74 in SMMC-7721 and HepG2 cells, respectively. The radiosensitivity of the cell lines under $\mathrm{CoCl}_{2}$ condition was further enhanced after the intervention of PX-478 (Figs. 2E and 2F). This effect was not observed under oxic condition. In addition, the radiosensitization effect of SSd was enhanced as SSd concentration increased, indicating that radiosensitivity was affected in a dose-dependent manner. Additionally, under hypoxic conditions $\left(1 \% \mathrm{O}_{2}, 5 \% \mathrm{CO}_{2}\right.$, and $94 \% \mathrm{~N}_{2}$ ),we also observed that SSd further decreases the SFs of radiation on SMMC-7721 cells or HepG2 cells (Figs. 2G and 2H).These results provided additional evidence that SSd may be a potent radiosensitizer in tumor cells.

\section{Effect of SSd on HIF-1 $\alpha$ and VEGF protein expression}

The expression levels of HIF- $1 \alpha$ protein in SMMC-7721 and HepG2 cells were examined to investigate the mechanism of radiosensitization of SSd on hematoma cells under hypoxic condition. The cells were incubated with $100 \mu \mathrm{M} \mathrm{CoCl}_{2}$ for $4 \mathrm{~h}$ in a serum-free medium. Low $\mathrm{HIF}-1 \alpha$ expression was detected in both cell lines before the $\mathrm{CoCl}_{2}$ treatment; this result is probably due to the rapid protein degradation under oxic conditions [25]. By contrast, the protein was expressed after the $\mathrm{CoCl}_{2}$ treatment (Figs. 3A and 3F). Radiation in the presence of $\mathrm{CoCl}_{2}$ killed fewer cells than in the absence of $\mathrm{CoCl}_{2}$ (Figs. 2B and 2D, $P<0.05$ ). The ability of radiation to kill tumor cells was significantly enhanced when SSd was added (Figs. 2B and 2D, $P<0.01$ ). HIF-1 $\alpha$ expression also decreased under $\mathrm{CoCl}_{2}$ conditions (Figs. 3B and 


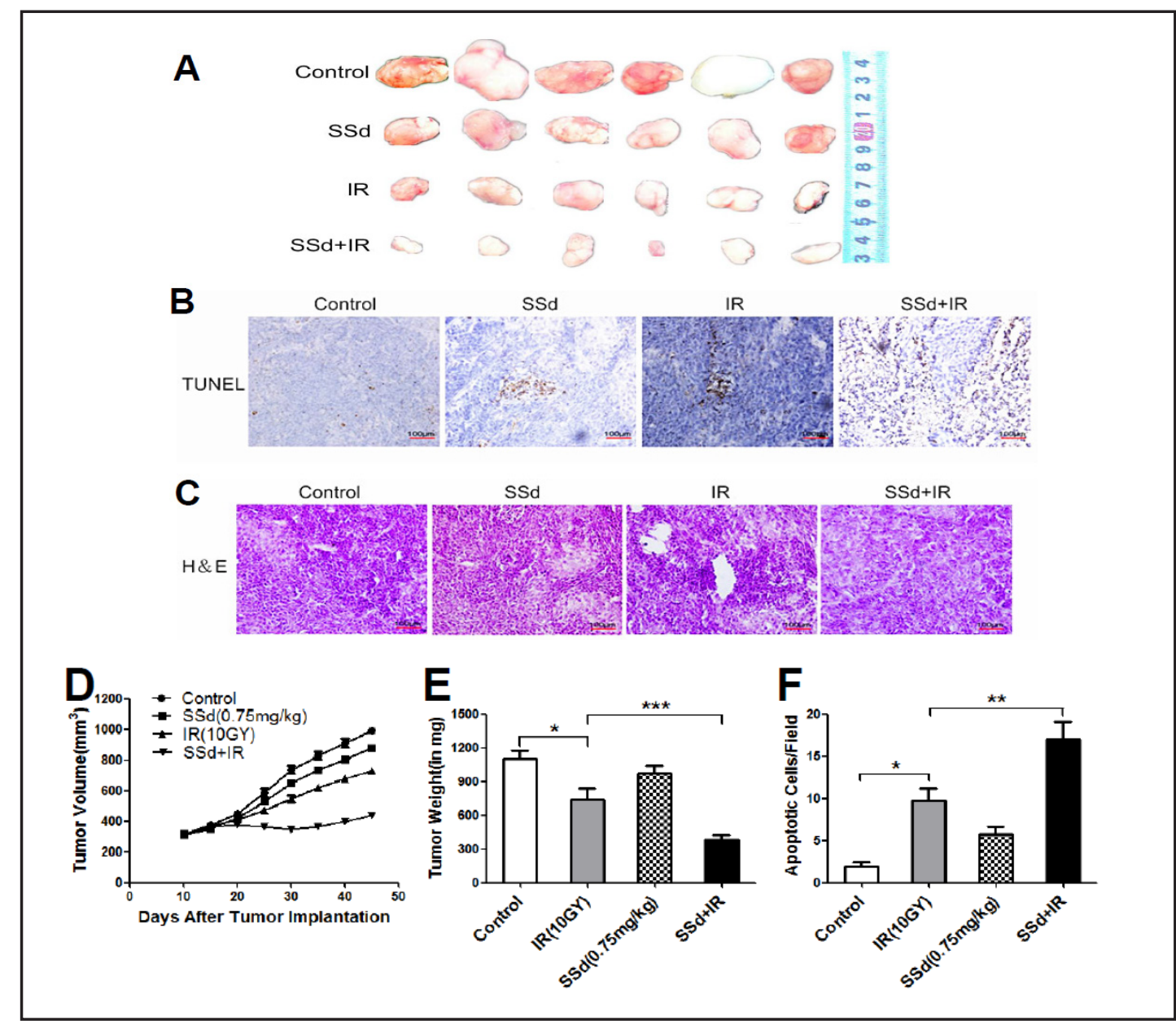

Fig. 5. Inhibitory effect of radiation plus SSd on tumor growth in nude mice xenograft. A. Representative photographs of xenografts in nude mice of each group at the end of the experimental period. B. TUNEL apoptosis of tumor sections from mice receiving different treatments. The apoptotic nuclei were stained dark brown. C. Hematoxylin and eosin staining (H\&E) of tumor tissues from mice receiving different treatments. The scale bar represents $100 \mu \mathrm{m}$. D. The changes of tumor volume after tumor implantation. E. Quantitation of tumor weight at the end of the experimental period. Data are presented as mean \pm SD $(n=6)$. F. Apoptosis analysis of tumor sections from mice receiving different treatments. Data are shown as mean $\pm \mathrm{SD} * \mathrm{P}<0.05$, ** $\mathrm{P}<0.01,{ }^{* * *} \mathrm{P}<0.001$.

$3 \mathrm{G}, P<0.01)$. After the cells were treated with PX-478, HIF-1 $\alpha$ expression decreased under $\mathrm{CoCl}_{2}$ conditions (Figs. 3A, 3B, 3F, and 3G, $P<0.01$ ), cell radioresistance disappeared, and cell apoptotic rate increased significantly (Figs. 1C and 1D, $P<0.01$ ). The lowest HIF-1 $\alpha$ expression was observed in the cells treated with PX-478 and SSd under $\mathrm{CoCl}_{2}$ condition. This result showed that HIF-1 $\alpha$ expression correlated negatively with cell radiosensitivity. In addition, we examined the VEGF expression of HIF-1 $\alpha$ downstream genes in SMMC7721 and HepG2 cells. The results showed that the upregulation or downregulation of VEGF expression is consistent with HIF-1 $\alpha$ expression after our intervention (Figs. 3A, 3E, 3 F, and 3J). Similarly, the expression levels of HIF-1 $\alpha$ protein in SMMC-7721 and HepG2 cells were significantly reduced under hypoxic conditions $\left(1 \% \mathrm{O}_{2}, 5 \% \mathrm{CO}_{2}\right.$, and $94 \% \mathrm{~N}_{2}$ ) (Fig. 4, $P$ $<0.01$ ). after treated with the combination of SSd and radiation. These results also suggested that $\mathrm{CoCl}_{2}$ or hypoxia-induced HIF-1 $\alpha$ overexpression functioned in cellular resistance to radiation, whereas SSd elicited an inhibitory effect on HIF-1 $\alpha$ expression in tumor cells under hypoxic conditions. 


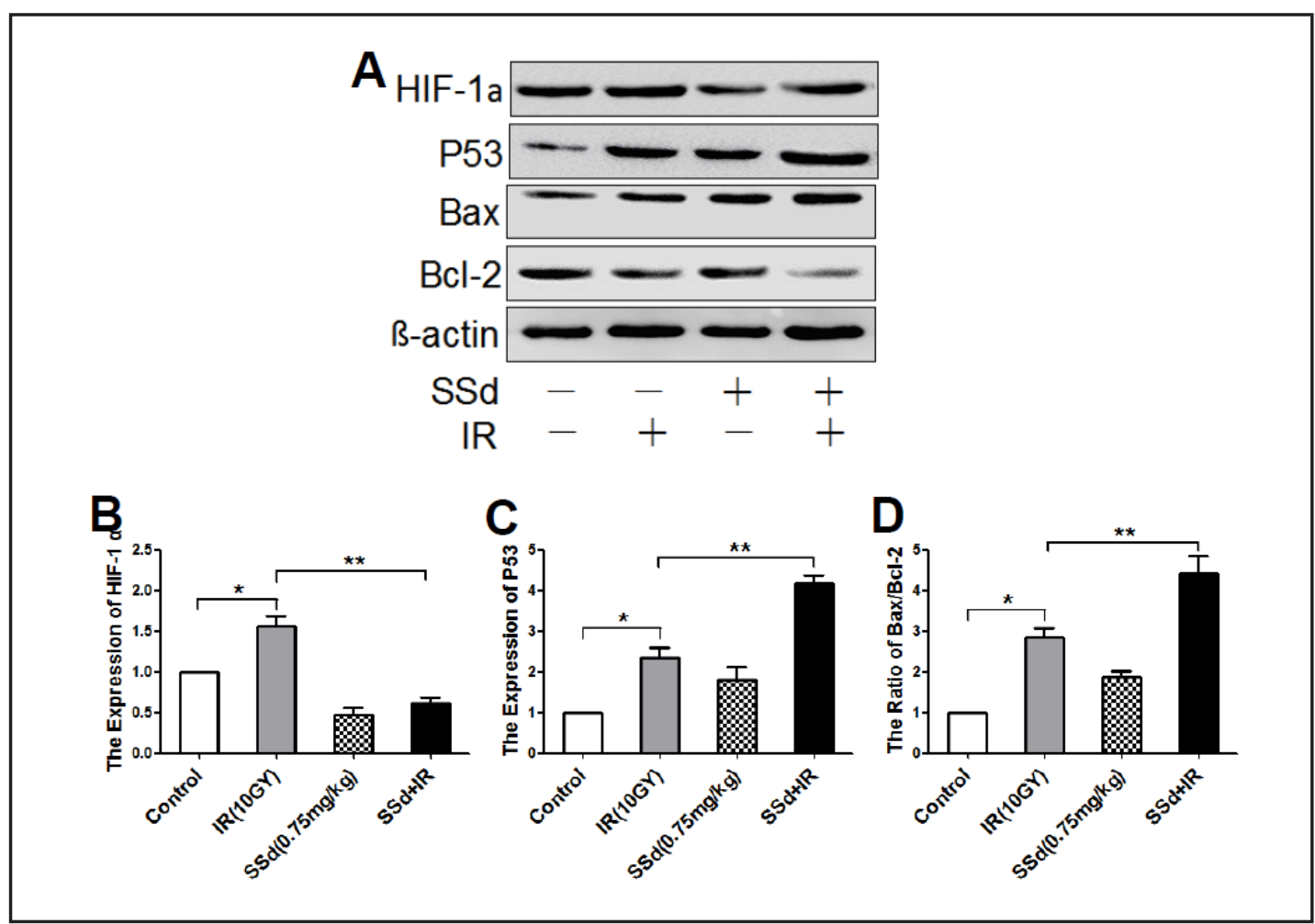

Fig. 6. Effects of different interventions on the expressions of HIF-1 $\alpha, \mathrm{P} 53$, Bax and Bcl-2 in xenografts. Cells were treated with vehicle control, SSd or radiation alone, or the combination of SSd and radiation. A. Western Blotting of HIF-1 $\alpha$, p53, Bax and Bcl-2 levels. B. Relative expression of HIF-1 $\alpha$. C. Relative expression of p53. D. Change of Bax/Bcl-2 ratio. Protein expression was normalized to that of $\beta$-actin. Data are shown as mean $\pm \mathrm{SD} * \mathrm{P}<0.05, * * \mathrm{P}<0.01$.

\section{Effect of SSd on radiation-induced $p 53$ and Bax/Bcl-2 expressions}

The tumor suppressor p53, also known as a "gatekeeper" protein, has a critical function in preventing cancer development by initiating cell apoptosis. P53 can induce the expression of pro-apoptotic Bax protein $[26,27]$ and suppress the expression of anti-apoptotic Bcl-2 [28, 29], resulting in apoptosis [30,31]. In our experiments, p53 level in the tumor cells did not change under $\mathrm{CoCl}_{2}$ condition (Figs. 3A and 3F). At 2 Gy of radiation, p53 protein expression increased in SMMC-7721 and HepG2 cells under oxic or $\mathrm{CoCl}_{2}$ conditions (Figs. 3A and 3F, $P$ $<0.01)$. A greater increase in p53 expression was induced in cells treated with the combined therapy under oxic (2.7-fold for SMMC-7721 and 2.5-fold for HepG2) and $\mathrm{CoCl}_{2}$ (3.5-fold for SMMC-7721 and 3.3-fold for HepG2) conditions (Figs. 3C and H). The Bax/Bcl-2 expression in SMMC-7721 cells increased by 3.3-fold under oxic condition and 4.0-fold under $\mathrm{CoCl}_{2}$ condition after the combined treatment was administered (Fig. 3D). In HepG2 cells, Bax/Bcl2 expression increased by 3.2-and 3.9-fold under oxic and $\mathrm{CoCl}_{2}$ conditions, respectively (Fig. 3I). These data provided evidence of a direct link between p53 expression and radiationinduced tumor cell apoptosis. These data also suggested that the radiosensitization effect of SSd may be associated with p53 expression under oxic and $\mathrm{CoCl}_{2}$ conditions.

Therapeutic efficacy of SSd in xenografted SMMC-7721 model in BALB/c nude mice

This experiment was performed to compare the efficacy of monotherapy with the combined therapy on tumor growth in vivo. SSd administered at a dose of $0.75 \mathrm{mg} / \mathrm{kg}$ showed a modest inhibition of tumor growth compared with the control group, resulting in $11 \%$ reduction in tumor weight. By contrast, radiation at a dose of 10 Gy significantly inhibited tumor growth, resulting in 33\% reduction in tumor weight at the end of the 


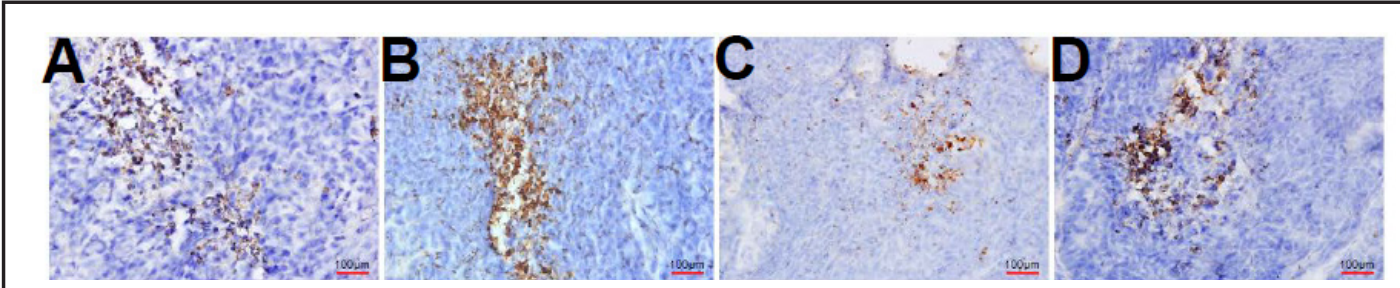

Fig. 7. Immunostaining analysis of pimonidazole expression in xenografts. A. Immunostaining of pimonidazole in the control. B. Immunostaining of pimonidazole in the radiation group. C. Immunostaining of pimonidazole in the SSd group. D. Immunostaining of pimonidazole in the combined group. E. Changes in pimonidazole intensity scores exposed to different interventions. The scale bar represents $100 \mu \mathrm{m}$. The pimonidazole-positive cells in the tumor sections are brown staining in cytoplasm. The mean scores were $5.76 \pm 1.89$ for the control tumors, $7.28 \pm 1.76$ for tumors treated with radiation alone, $3.48 \pm 0.96$ for the tumors receiving SSd, and $3.75 \pm 0.85$ for tumors receiving both SSd and radiation. The combined group presented significantly reduced hypoxic fractions compared with the control group. Data are shown as mean $\pm \mathrm{SD} * \mathrm{P}<0.05$, ${ }^{* *} \mathrm{P}<0.01$.

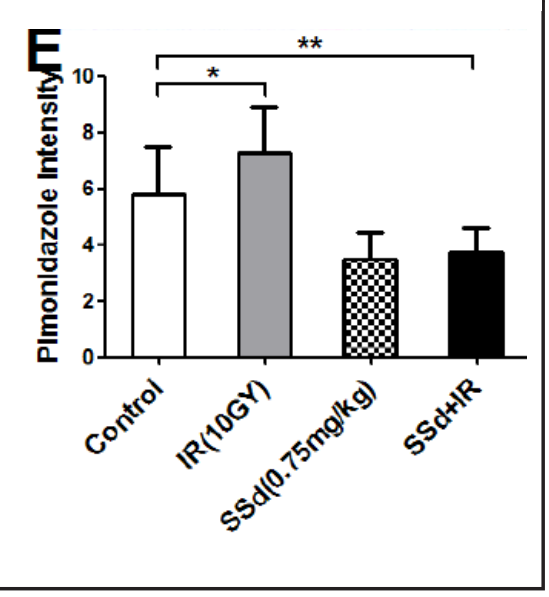

experiment (Figs. 5A and 5E, $P<0.05$ ). The combined SSd and radiation also resulted in $66 \%$ reduction in tumor weight (Fig. 5E, $P<0.001$ ). Tumor growth slowed down and reached the minimum level at $13 \mathrm{~d}$ after irradiation; the tumor volume obtained at this time was only $47 \%$ of the tumor volume of the control group (Fig. 5D). The experiment was terminated three weeks after the final injection of SSd. The mice were sacrificed and the tumor tissues were examined to determine HIF-1 $\alpha$ expression. HIF-1 $\alpha$ expression increased in the cells treated with radiation alone compared with that in the control group; by contrast, HIF-1 $\alpha$ expression decreased in the cells treated with either SSd alone or the combined regimen (Figs. 6A and 6B, $P<0.05$ ). In addition, we further assess tumor hypoxia by performing pimonidazole immunohistochemistry of tumor tissue using a Hypoxyprobe ${ }^{\mathrm{TM}}-1$ Kit. The results also showed that after treated with the combination of SSd and radiation, the number of pimonidazole positive cells and the staining intensity was significantly lower than the control group. (Fig.7, $P<0.01$ ).

TUNEL assay was conducted; p53, Bax, and Bcl-2 expressions were examined. As expected, the combined therapy significantly induced apoptosis as shown by an increased number of apoptotic cells compared with the monotherapies (Figs. 5B and 5F, $P<0.01$ ). Increased p53 expression and ratio of Bax/Bcl-2 also indicated that apoptosis was significantly induced by the combined therapy (Figs. 6A, 6C, and 6D, $P<0.01$ ). H\&E also revealed that tumors from the mice subjected to the combined therapy exhibited a higher apoptotic rate than those from the mice treated with either of the monotherapies (Fig. 5C).

\section{Discussion}

HIF-1 $\alpha$ overexpression in human cancer cells is correlated with the poor response of tumor cells to radiotherapy and poor prognosis [10, 32]. Therefore, HIF-1 $\alpha$ is considered as a potential therapeutic target and HIF- $1 \alpha$ inhibition is a promising strategy to treat cancer. The in vitro experiments in this study showed that SSd could enhance the radiosensitivity of SMMC-7721 and HepG2 cell lines irradiated under oxic and hypoxic conditions. SSd could inhibit the expressions of HIF- $1 \alpha$ and VEGF more effectively in cells irradiated under hypoxic condition than under oxic condition. The cells exposed to the combined treatment exhibited cell apoptosis with a higher Bax/Bcl ratio than those treated with either SSd or 
radiation alone. The combined treatment also increased p53 expression in cells. The in vivo experiments revealed that the combined treatment more evidently inhibited tumor growth than either of the monotherapies. Tumor growth was significantly slowed down and cell apoptosis occurred at a high rate after the combined treatment. The remarkable decrease in HIF-1 $\alpha$ expression in the cells treated with SSd alone or SSd and radiation indicated that SSd possibly inhibited HIF-1 $\alpha$ expression in tumor cells and enhanced tumor radiosensitivity.

SSd can increase radiation-induced apoptosis of tumor cells and reduce cell colony formation under oxic conditions. Under oxic conditions, HIF- $1 \alpha$ and VEGF expression levels were low in the tumor cells; these expressions increased to a certain extent after irradiation. Although SSd and radiation were combined, a significant change was not observed in HIF-1 $\alpha$ and VEGF expression. This result indicated that the increase in radiosensitivity caused by SSd under oxic conditions is unlikely a result of inhibited HIF- $1 \alpha$ and VEGF expressions. This finding is consistent with that in other studies, in which SSd involves many other mechanisms in tumor growth inhibition $[18,19]$. The molecular mechanisms of the radiosensitization effect of SSd under oxic condition need further investigation. In the present study, HIF$1 \alpha$ and VEGF expression levels in tumor cells under hypoxic conditions were significantly higher. The cells also showed higher radioresistance level; the inhibition of radiation-induced apoptosis and colony formation was significantly reduced. SSd induced a decrease in HIF- $1 \alpha$ and VEGF expressions; the ability of radiation to kill tumor cells when combined with SSd was significantly enhanced. In addition, PX-478 inhibited tumor growth more effectively as indicated by a higher HIF- $1 \alpha$ activity under $\mathrm{CoCl}_{2}$ condition than under oxic condition. This finding is consistent with that in previous reports [33-35]. With the co-intervention of PX478 and SSd, radioresistance of tumor cells under $\mathrm{CoCl}_{2}$ conditions decreased significantly as HIF- $1 \alpha$ and VEGF expressions in the cells further decreased. The ability of radiation to kill tumor cells was enhanced. However, this enhancement was not observed under oxic conditions. Therefore, the radiosensitization effect of SSd under hypoxic conditions could be attributed to the inhibition of HIF-1 $\alpha$ expression.

The precise mechanism of radiosensitivity enhancement in cells pre-incubated with SSd remains unclear. The p53 protein is likely the most thoroughly studied tumor suppressor involved in preventing cancer development by regulating numerous cellular processes that participate in DNA repair after genotoxic stress $[36,37]$. In addition to the ability of p53 to initiate apoptosis of damaged cells, the level of p53 is influenced by radiation treatment $[38,39]$. A study has found that pre-irradiation treatment with adenovirus-mediated p53 may significantly increase the radiosensitivity of tumor cells [40]. Anti-cancer functions of p53 are largely demonstrated by an apoptosis-inducing ability. P53 can also induce the expression of pro-apoptotic Bcl-2 family members, such as Bax, Bid, Noxa, and Puma [26, 27], which activates caspase-3, in the apoptosis pathway [30, 31]. By contrast, p53 suppresses the expression of anti-apoptotic genes such as Bcl-2, Bcl-xL, and survivin [28, 29].

We found in our experiments that $\mathrm{CoCl}_{2}$ alone has no effect on p53 expression in tumor cells, whereas radiation can induce the increase in p53 protein level in oxic and $\mathrm{CoCl}_{2}$-treated cells. Equal doses of radiation produce a higher survival rate in $\mathrm{CoCl}_{2}$-treatedcells than in oxic cells. This result corresponds to the changes observed in p53 levels after equal doses of radiation were administered, in which p53 levels are significantly increased in oxic cells but not in $\mathrm{CoCl}_{2}$-treated cells. P53 is known to have a major function in the induction of apoptosis as a result of radiation treatment $[30,38]$. Downstream effectors of p53 were examined after irradiation. The results showed a more significant upregulation of proapoptotic Bax and $\mathrm{Bax} / \mathrm{Bcl}-2$ ratio under oxic condition than under $\mathrm{CoCl}_{2}$ condition. By contrast, the expression of Bcl-2 is more remarkably downregulated under oxic condition and tumor cell apoptosis rate is significantly higher. Ayelet et al. [41] reported similar results of TK6 human lymphoblastoid cells. These findings suggested that the induction of p53 is a radiation-specific stimulus in oxic and $\mathrm{CoCl}_{2}$-treated tumor cells; the radiosensitizing effect of SSd on hepatoma cells may be closely associated with p53.

The high expression of HIF- $1 \alpha$ under hypoxic conditions may inhibit p53 expression, resulting in the radioresistance of tumor cells $[42,43]$. Our in vitro study showed that the 
HIF-1 $\alpha$ expression decreased as p53 inhibition was relieved under $\mathrm{CoCl}_{2}$ condition after the combined therapy was administered. The in vivo experiment also revealed a decrease in HIF- $1 \alpha$ and pimonidazole expression and an increase in p53 levels after the intervention of SSd in radiation treatment. These findings suggested that the radiosensitization effect of SSd under hypoxic conditions may be observed by enhancing p53 expression caused by the inhibition of HIF-1 $\alpha$ expression.

In conclusion, the radiosensitization effect of SSd on hepatoma cells was demonstrated for the first time. The mechanism of this effect may involve the inhibition of HIF-1 $\alpha$ expression by SSd in hypoxic tumor cells. Our experimental data suggested that the radiosensitization effect of SSd on hepatoma cells may be closely related to the p53 expression in cells, but this association should be further verified. The findings in this study indicated that SSd may be a potential sensitizer in liver cancer radiotherapy.

\section{Conflict of Interest}

All authors have no financial and personal relationships with other people or organizations that may inappropriately influence (bias) their work.

\section{Acknowledgments}

This work was supported by the National Natural Science Foundation of China (No. 30973810).

\section{References}

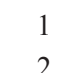

2

$>3$

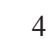

4 Gastroenterology 2007;132:2557-2576. comparison of treatment combinations with and without radiotherapy for hepatocellular carcinoma with portal vein and/or inferior vena cava tumor thrombus. Int J Radiation Oncology Boil Phys 2005;61:432443.

5 Merle P, Mornex F, Trepo C: Innovative therapy for hepatocellular carcinoma: Three-dimensional high-dose photon radiotherapy. Cancer Lett 2009;286:129-133

-6 Hawkins MA, Dawson LA: Radiation therapy for hepatocellular carcinoma: from palliation to cure. Cancer 2006;106:1653-1663.

7 Bache M, Kappler M, Said HM, Staab A, Vordermark D: Detection and specific targeting of hypoxic regions within solid tumors: current preclinical and clinical strategies. Curr Med Chem 2008;15:322-338.

8 Dewhirst MW, Cao Y, Moeller B: Cycling hypoxia and free radicals regulate angiogenesis and radiotherapy response. Nature Rev Cancer 2008;8:425-437.

-9 Semenza GL: Targeting HIF-1 for cancer therapy. Nat Rev Cancer 2003;3:721-732.

10 Aebersold DM, Burri P, Beer KT, Laissue J, Djonov V, Greiner RH, Semenza GL: Expression of hypoxiainducible factor-1alpha: a novel predictive and prognostic parameter in the radiotherapy of oropharyngeal cancer. Cancer Res 2001;61:2911-2916.

- 11 Singh-Gupta V, Zhang H, Banerjee S, Kong D, Raffoul JJ, Sarkar FH, Hillman GG: Radiation-induced HIF-1 $\alpha$ lpha cell survival pathway is inhibited by soy isoflavones in prostate cancer cells. Int J Cancer 2009;124:1675-1684. 
12 Harada H, Kizaka-Kondoh S, Li G, Itasaka S, Shibuya K, Inoue M, Hiraoka M: Significance of HIF-1- active cells in angiogenesis and radio- resistance. Oncogene 2007;26:7508-7516.

13 Staab A, Loeffler J, Said HM, Diehlmann D, Katzer A, Beyer M, Fleischer M, Schwab F, Baier K, Einsele H, Flentje M, Vordermark D: Effects of HIF-1 inhibition by chetomin on hypoxia-related transcription and radio- sensitivity in HT 1080 human fibrosarcoma cells. BMC Cancer 2007;7:213.

14 Lu CN, Yuan ZG, Zhang XL, Yan R, Zhao YQ, Liao M, Chen JX: Saikosaponin a and its epimer saikosaponin-d exhibit anti- inflammatory activity by suppressing activation of NF- $\mathrm{BB}$ signaling pathway. Int Immunopharmacol 2012;14:121-126.

-15 Hattori T, Nishimura H, Kase Y, Takeda S: Saireito and saikosaponin D prevent urinary protein excretion via glucocorticoid receptor in adrenalectomized WKY rats with heterologous-phase anti-GBM nephritis. Nephron Physiol 2008;109:19-27.

16 Fan J, Li X, Li P, Li N, Wang T, Shen H, Siow Y, Choy P, Gong Y: Saikosaponin-d attenuates the development of liver fibrosis by preventing hepatocyte injury. Biochem Cell Biol 2007;85:189-195.

$\checkmark 17$ Wang BF, Cheng YA, Dang SS: Angiogenesis inhibitory effect of saikosaponin-d on chicken embryo. Zhongguo Zhong Xi Yi Jie He Za Zhi 2009;29:425-429.

18 Hsu YL, Kuo PL, Chiang LC, Lin CC: Involvement of p53, nuclear factor kappaB and Fas/Fas ligand in induction of apoptosis and cell cycle arrest by saikosaponin $\mathrm{d}$ in human hepatoma cell lines. Cancer Lett 2004;213:213-221.

19 Wang Q, Zheng XL, Yang L, Shi F, Gao LB, Zhong YJ, Sun H, He F, Lin Y, Wang X: Reactive oxygen speciesmediated apoptosis contributes to chemosensiti- zation effect of saikosaponins on cisplatin-induced cytotoxicity in cancer cells. J Exp Clin Cancer Res 2010;29:159.

20 Yang W, Sun T, Cao J, Fan S: Hypoxia-Inducible Factor-1 $\alpha$ Downregulation by Small Interfering RNA Inhibits Proliferation, Induces Apoptosis, and Enhances Radiosensitivity in Chemical Hypoxic Human Hepatoma SMMC-7721 Cells. Cancer Biother Radiopharm 2011;26:565-571.

-21 Ji Z, Yang G, Shahzidi S, Tkacz-Stachowska K, Suo Z, Nesland JM, Peng Q: Induction of hypoxia-inducible factor-1alpha overexpression by cobalt chloride enhances cellular resistance to photodynamic therapy. Cancer Lett 2006;244:182-189.

22 Jiang H, Wang H, Tan Z, Hu S, Wang H, Shi B, Yang L, Li P, Gu J, Wang H, Li Z: Growth suppression of human hepatocellular carcinoma xenografts by a monoclonal antibody $\mathrm{CH} 12$ directed to epidermal growth factor receptor variant III. J Biol Chem 2011;286:5913-5920.

23 Wang H, Jiang H, Zhou M, Xu Z, Liu S, Shi B, Yao X, Yao M, Gu J, Li Z: Epidermal growth factor receptor vIII enhances tumorigenicity and resistance to 5-fluorouracil in human hepatocellular carcinoma. Cancer Lett 2009;279:30-38.

24 Nordsmark M, Loncaster J, Aquino-Parsons C, Chou SC, Ladekarl M, Havsteen H, Lindegaard JC, Davidson SE, Varia M, West C, Hunter R, Overgaard J, Raleigh JA.Measurements of hypoxia using pimonidazole and polarographic oxygen-sensitive electrodes in human cervixcarcinomas. Radiother Oncol 2003;67:35-44.

25 Dery MA, Yonuschot G, Winterson BJ: The effects of manually applied intermittent pulsation pressure to rat ventral thorax on lymph transport. Lymphology 2000;33:58-61.

-26 Chipuk JE, Kuwana T, Bouchier-Hayes L, Droin NM, Newmeyer DD, Schuler M, Green DR: Direct activation of Bax by p53 mediates mitochondrial membrane permeabilization and apoptosis. Science 2004;303:10101014.

27 Vousden KH, Prives C: Blinded by the light: the growing complexity of p53. Cell 2009;137:413-431.

-28 Laptenko 0, Prives C: Transcriptional regulation by p53: one protein Many possibilities. Cell Death Differ 2006;13:951-961.

29 Mihara M, Erster S, Zaika A, Petrenko 0, Chittenden T, Pancoska P, Moll UM: P53 has a direct apoptogenic role at the mitochondria. Mol Cell 2003;11:577-590.

30 Henry H, Thomas A, Shen Y, White E: Regulation of the mitochondrial checkpoint in p53-mediated apoptosis confers resistance to cell death. Oncogene 2002;21:748-760.

31 Menendez D, Inga A, Resnick MA: The expanding universe of p53 targets. Nat Rev Cancer 2009;9:724-737.

- 32 Koukourakis MI, Bentzen SM, Giatromanolaki A, Wilson GD, Daley FM, Saunders MI, Dische S, Sivridis E, Harris AL: Endogenous markers of two separate hypoxia response pathways (hypoxia inducible factor 2a and carbonic anhydrase 9) are associated with radiotherapy failure in head and neck cancer patients recruited in the CHARTrandomized trial. J Clin Oncol 2006;24:727-735. 
-33 Rapisarda A, Uranchimeg B, Scudiero DA, Selby M, Sausville EA, Shoemaker RH, Melillo G: Identification of small molecule inhibitors of hypoxia- inducible factor 1 transcriptional activation pathway. Cancer Res 2002;62:4316-4324.

-34 Koh MY, Spivak-Kroizman T, Venturini S, Welsh S, Williams RR, Kirkpatrick DL, Powis G: Molecular mechanisms for the activity of PX-478, an antitumor inhibitor of the hypoxia-inducible factor- $1 \mathrm{a}$. Mol Cancer Ther 2008;7:90-100.

-35 Zhong H, Willard M, Simons J: NS398 reduces hypoxia-inducible factor (HIF)-1 $\alpha$ and HIF-1 activity: multiple-level effects involving cyclooxygenase-2 dependent and independent mechanisms. Int J Cancer 2004;112:585-595.

36 Vousden KH: p53: death star. Cell 2000;103:691-694.

-37 Ferguson DO, Alt FW: DNA double strand break repair and chromosomal translocation: lessons from animal models. Oncogene 2001;20:5572-5579.

38 Liang L, Shao C, Deng L, Mendonca MS, Stambrook PJ, Tischfield JA: Radiation-induced genetic instability in vivo depends on p53 status. Mutat Res 2002;502:69-80.

-39 Kastan MB, Zhan Q, El-Deiry WS, Carrier F, Jacks T, Walsh WV, Plunkett BS, Vogelstein B, Fornace AJ Jr: A mammalian cell cycle checkpoint pathway utilizing p53 and GADD45 is defective in ataxia-telangiectasia. Cell 1992;71:587-597.

40 Sah NK, Munshi A, Nishikawa T, Mukhopadhyay T, Roth JA, Meyn RE: Adenovirus-mediated wild-type p53 radiosensitizes human tumor cells by suppressing DNA repair capacity. Mol Cancer Ther 2003;2:12231231.

-41 Samuni AM, Kasid U, Chuang EY, Suy S, Degraff W, Krishna MC, Russo A, Mitchell JB: Effects of Hypoxia on Radiation-Responsive Stress- Activated Protein Kinase, p53, and Caspase 3 Signals in TK6 Human Lymphoblastoid Cells. Cancer Res 2005;65:579-586.

-42 Sendoel A, Kohler I, Fellmann C, Lowe SW, Hengartner MO: HIF-1 antagonizes p53-mediated apoptosis through a secreted neuronal tyrosinase. Nature 2010;465:577-583.

43 Lee KB, Kim KR, Huh TL, Lee YM: Proton induces apoptosis of hypoxic tumor cells by the p53-dependent and p38/JNK MAPK signaling pathways. Int J Oncol 2008;33:1247-1256. 